\title{
Valuing defaultable bonds: an excursion time approach
}

\author{
Martina Nardon \\ Dipartimento di Matematica Applicata - Università Ca' Foscari di Venezia \\ Dorsoduro 3825/E - 30123 Venezia, Italy \\ mnardon@unive.it
}

\begin{abstract}
Recently there has been some interest in the credit risk literature in models which involve stopping times related to excursions. The classical Black-Scholes-Merton-Cox approach postulates that default may occur, either at or before maturity, when the firm's value process falls below a critical threshold. In the excursion approach the duration of default, the time period from the financial distress announcement through its resolution, is explicitly modeled. In this contribution, we provide a review of the literature on excursion time models of credit risk. Moreover, we examine the effects on credit spreads structure of different specifications of the event that triggers default.

Keywords. Credit risk, structural models, excursion approach, default threshold, default probability.
\end{abstract}

M.S.C. Classification: 60G40, 60J60, 65C20, $91 \mathrm{~B} 28$.

J.E.L. Classification: C15, C63, G12, G13.

\section{Introduction}

Default risk is a crucial determinant of the values of bonds. A variety of approaches have been proposed in the literature with the aim of evaluating defaultable debt and trying to better explain the process that drives default.

In the class of structural credit risk models, based on the works of Black and Scholes (1973) [3], Merton (1974) [24], and Black and Cox (1976) [2], default occurs, either at or before maturity, if the firm's value is below a certain level, which represents liabilities (or part of them), or some safety covenant, which provides debt-holders with the right to force firm into bankruptcy.

First-passage time models ${ }^{1}$, introduced by Black and Cox (1976) [2], allow for firm bankruptcy prior to maturity. More precisely, the time of default is

\footnotetext{
${ }^{1}$ A selection of important contributions in this class includes, but it is not limited to, Black and Cox (1976) [2], Kim et al. (1993) [20], Nielsen et al. (1993) [27], Shimko et al. (1993) [31], Longstaff and Schwartz (1995) [23], Bryis and de Varenne (1997) [4], Cathcart and El-Jahel (1998) [6], Saá-Requejo, Santa-Clara (1999) [30], Taurén (1999) [32], Collin-Dufresne and Goldstein (2001) [8], Hsu et al. (2003) [16], and Hui et al. (2003) [18].
} 
the first instant at which the firm's value process (or another relevant process) reaches a critical threshold, called the default boundary. Such a boundary can be assumed either deterministic (a constant threshold or a time-varying barrier) or stochastic. It may even be determined endogenously as the solution of an optimal decision problem ${ }^{2}$.

In practice, once a firm has encountered financial distress, it may be either reorganized or liquidated. In first-passage time modeling of credit risk, no distinction is made between the time at which a firm enters bankruptcy and the time at which it is either liquidated or the reorganization plan is accepted and the firm exits bankruptcy. In the excursion time approach, the time spent in default (from the bankruptcy announcement through its resolution) is explicitly modeled.

Some empirical works (among which we mention Eberhart et al. (1999) [10] and Helwege (1999) [15]) investigate firms emerging from bankruptcy, and find that the time period spent in financial distress is in some cases of a few months, on average it ranges from two to three years, and that longest defaults lasted up to several years.

Recently, there has been some interest in the credit risk literature in models which involve stopping times related to excursions. Within this class of models, we may cite the contributions of Galai et al. (2003) [12], Moraux (2003) [25], Paseka (2003) [28], François and Morellec (2004) [11], Broadie et al. (2004) [5], and $\mathrm{Yu}$ et al. (2004) [33].

In this contribution, we provide a review of excursion time methods for credit risk. In particular, we study an approach that accounts for the severity of the financial distress. The main focus is on the definition of the timing of default. We then examine the effects on credit spreads structure of different specifications of the event that triggers default within a particular structural model.

The remainder of the paper is organized as follows. Section 2 introduces the general valuation framework. Section 3 briefly reviews the first-passage approach. Section 4 is dedicated to the excursion approach. Section 5 presents an excursion time model in which the severity of the financial distress is expressly modeled. In Section 6 some results of the simulation analysis are presented. Section 7 concludes.

\section{The valuation framework}

In credit risk models, important features derive from the crucial assumptions made about the following elements: the dynamics of the process that governs default, the default boundary, the (possibly random) variable that describes the recovery value upon default, and the dynamics of the default-free interest rate.

We need to define a value process $X$, whose passage through a prespecified boundary signals a credit event, such as financial distress or a change in creditworthiness.

\footnotetext{
${ }^{2}$ See, e.g., Leland (1994) [21], and Leland and Toft (1996) [22].
} 
Formally, let us consider a continuous time model on the time horizon $[0, \bar{T}]$. Assume that a probability space $(\Omega, \mathcal{F}, \mathbb{P})$ is equipped with a filtration $\left(\mathcal{F}_{t} ; t \geq\right.$ $0)$, provided some technical conditions, where $\mathcal{F}_{t}$ represents the information set at time $t$.

In a contingent claims framework (provided the usual assumptions of perfect and frictionless markets), consider a firm with a single issue of debt in the form of a zero-coupon bond (hereafter ZCB) promising to pay the face value (which is assumed to be one euro) at the maturity date $T$. Let $p(t, T)$ and $v(t, T)$ denote the price at time $t \leq T$ of default-free and defaultable ZCBs, respectively, with maturity T. Assume, moreover, that ZCBs are traded for all maturities. Both defaultable and default-free bonds markets are assumed to be arbitrage-free, so there exists an equivalent martingale measure $\mathbb{Q}^{3}$.

In this setting, consider a stochastic process $X=\left(X_{t}\right)_{t \geq 0}$ (continuous and adapted), which describes the firm's value (or another relevant firm's feature, such as assets value, solvency ratio, cash balances, etc.). Then, the information set at time $t$ will be $\mathcal{F}_{t}=\sigma\left(X_{u} ; u \leq t\right)$. In first passage and excursion approaches, we need some assumptions on the default boundary $H=\left(H_{t}\right)_{t \in[0, T]}$. Note that, when the default boundary is driven by a stochastic process, the information set is $\mathcal{F}_{t}=\sigma\left(X_{u}, H_{u} ; u \leq t\right)$.

At this stage, no assumption is made about the spot rate dynamics. Let $r=$ $\left(r_{t}\right)_{t \geq 0}$ denote the default-free spot interest rate, $r$ will be either deterministic (constant in the simplest case, or a time-varying function) or governed by some stochastic process. In this latter case, $r$ will depend on a Wiener process, possibly correlated with the process $X$ governing default.

\section{$3 \quad$ First-passage time approach}

In first-passage time models, the time of default is a random variable whose distribution is that of the first-hitting time of the value process $X$ to the default boundary $\left(H_{t}\right)_{t \in[0, T]}$. Formally,

$$
\tau^{H}=\inf \left\{t \in[0, T): X_{t}=H_{t}\right\},
$$

with $\inf \emptyset=\infty$.

The random time $\tau^{H}$ is a predictable stopping time ${ }^{4}$. This means that default is not completely a surprise, but can be anticipated by observing the path taken by the process $X$.

Note that, at maturity of the debt default has to be specified carefully to avoid inconsistencies (see Giesecke (2004) [13]): at time $T$, typically default occurs if

\footnotetext{
3 This probability measure may not be unique (see Jarrow and Protter (2004) [19]).

${ }^{4}$ Given a complete probability space $(\Omega, \mathcal{F}, \mathbb{P})$, furnished with a filtration $\left(\mathcal{F}_{t}\right)_{t \geq 0}$, a random variable $\tau: \Omega \rightarrow[0, \infty]$ is a stopping time if the event $\{\omega \in \Omega: \tau(\omega) \leq$ $t\} \in \mathcal{F}_{t}$, for every $t \geq 0$.

A stopping time $\tau$ is predictable if there exists a sequence of stopping times $\left(\tau_{n}\right)_{n \geq 1}$ such that $\tau_{n}$ is increasing, $\tau_{n}<\tau$ on $\{\tau>0\}$, for all $n$, and $\lim _{n \rightarrow \infty} \tau_{n}=\tau$ a.s. The sequence $\left(\tau_{n}\right)$ is said to announce $\tau$ (See Protter (2004) [29]).
} 
assets value is less than the face value of the debt $F$. Let $\tau_{T}$ denote the random variable defined as follows

$$
\tau_{T}= \begin{cases}T & \text { if } X_{T} \leq F \\ \infty & \text { otherwise }\end{cases}
$$

Then the default time $\tau^{*}$ may be defined as

$$
\tau^{*}=\min \left\{\tau^{H}, \tau_{T}\right\}
$$

and the default probability is given by $\mathbb{Q}_{t}\left[\tau^{*} \leq T\right]=\mathbb{E}_{t}^{\mathbb{Q}}\left[\mathbf{1}_{\left\{\tau^{*} \leq T\right\}}\right]$, where $\mathbb{E}_{t}^{\mathbb{Q}}(\cdot)$ denotes expectation under the probability measure $\mathbb{Q}^{5}$, conditional on no current default, and $\mathbf{1}_{A}$ is the indicator function of $A$.

The default probability can be evaluated analytically in some special cases. As an example, assume a constant interest rate $r>0$, and let $X$ satisfy the following stochastic differential equation

$$
\frac{d X_{t}}{X_{t}}=r d t+\sigma d W_{t}, \quad X_{0}>0
$$

where $\sigma>0$, and $W=\left(W_{t}\right)_{t \geq 0}$ is a standard Wiener process under the risk neutral measure. If we assume that the default threshold is constant, $H_{t}=H$ for every $t \in[0, T]$, and that the recovery value upon default is constant and equal to the boundary value $H$, then we have the following well known results ${ }^{6}$ for the value at time $t$ of a defaultable ZCB expiring at time $T$ and the default probability $\mathbb{Q}_{t}\left(\tau^{H} \leq T\right)$,

$$
v(t, T)=H e^{-r(T-t)} \mathbb{Q}_{t}\left(\tau^{H} \leq T\right)+e^{-r(T-t)}\left[1-\mathbb{Q}_{t}\left(\tau^{H} \leq T\right)\right],
$$

where

$$
\begin{aligned}
\mathbb{Q}_{t}\left(\tau^{H} \leq T\right)=\Phi\left(h_{1}\right)+\left(\frac{X_{t}}{H}\right)^{-2 \frac{\left(r-\sigma^{2} / 2\right)}{\sigma^{2}}} \Phi\left(h_{2}\right), \\
h_{1,2}=\frac{-\log \left(X_{t} / H\right) \mp\left(r-\sigma^{2} / 2\right)(T-t)}{\sigma \sqrt{T-t}},
\end{aligned}
$$

and $\Phi(\cdot)$ is the standard normal distribution function.

In general, no closed form solutions are known for the default probability (in particular when some simplifying assumptions regarding default-free interest rate dynamics are relaxed) and numerical computations are required. Monte Carlo simulation can be used in order to estimate the default probability and the average time of default.

\footnotetext{
${ }^{5}$ We will write simply $\mathbb{E}_{t}(\cdot)$ shorthand for $\mathbb{E}_{t}^{\mathbb{Q}}(\cdot)$.

${ }^{6}$ See e.g. Bielecki and Rutkowski (2002) [1], and Giesecke (2004) [13].
} 


\section{Excursion time approach}

First-passage approach does not distinguish between the time at which a firm enters bankruptcy and the time at which it is either liquidated or reorganized. In the classical Black-Scholes-Merton-Cox approach, a firm defaults when its assets are too low according to some criterion, and it is immediately liquidated: this definition of default no longer reflects economic reality. Bankruptcy laws commonly grant an extended time period for the resolution of financial distress. In practice, upon default creditors have the right (not the obligation) to exercise their claims. When some debt covenant is violated, debt-holders could defer the decision of forcing firm into liquidation. They may try to enforce their position in order to interfere in the decisions of the debtor and require, for instance, troubled firm to maintain a certain financial ratio. While, in case of failure, the bankruptcy procedure could be accelerated.

In the excursion time approach, the duration of the default is a key element of the model. Recently, a few models have been proposed in the credit risk literature which involve excursions of a value process in a certain region and related stopping times. The excursion or Parisian time can be defined as the first instant when a relevant stochastic process (typically a Brownian motion) spends a given amount of time (the window) consecutively beyond a certain barrier. This stopping time has been used by Chesney et al. (1997) in order to define Parisian barrier options ${ }^{7}$.

Compared to the models based on first-passage times, in which a simple hitting of the barrier causes default with immediate liquidation of the firm, this formulation does not suffer from a threshold effect ${ }^{8}$. Even if the value process has crossed the barrier, default has not necessarily occurred yet. Unlike first-passage time approach, excursion approach allows for a non-absorbing state of default, and seems attractive in order to assessing default risk when a grace period is granted.

Excursion times could be useful in modeling real situations when delays, between the indication of financial distress and its resolution are involved. The delay could be a parameter exogenously specified, possibly prescribed by a debt covenant, or which has to be estimated from market data. As an alternative, the duration of the default (together with the default boundary) could also be endogenously determined (see Paseka (2003) [28]).

In the following, we assume that the delay is constant and denote it by $\Delta$. For simplicity, and without loss of generality, let us consider a constant default boundary, $H$. Suppose also $X_{0}>H$.

Let

$$
\tau_{t}^{H}=\sup \left\{u \leq t: X_{u}=H\right\}
$$

be the last time, before $t$, that the process $X$ hits the threshold $H$.

\footnotetext{
${ }^{7}$ Parisian options are financial derivatives for which the barrier in- or out-feature is activated when the underlying price process spent continuously a certain amount of time beyond the barrier.

8 See Haber et al. (1999) [14].
} 
Define the excursion (or Parisian) time

$$
\tau_{\Delta}^{H}=\inf \left\{t \geq 0:\left(t-\tau_{t}^{H}\right) \mathbf{1}_{\left\{X_{t} \leq H\right\}} \geq \Delta\right\} .
$$

The default time is defined as the random time $\tau_{\Delta}^{H}$ and the default probability is $\mathbb{Q}\left[\tau_{\Delta}^{H} \leq T\right]=\mathbb{E}\left[\mathbf{1}_{\left\{\tau_{\Delta}^{H} \leq T\right\}}\right]$.

Le us observe that the default probability in the excursion approach lies between two limit cases. When the time window $\Delta$ is zero, we recover the simple first-passage case, while when $\Delta$ is very large and approaches $T$, one has to check for default only at maturity of the debt, hence the probability of default reduces to that in the Black-Scholes-Merton model.

Define now the random quantity, called the distress clock,

$$
\Delta_{t}=t-\tau_{t}^{H} \quad t \in[0, T] .
$$

$\Delta_{t}$ is the length of time the process $X$ has spent below the default barrier in the current excursion ${ }^{9}$.

Starting from $X_{0}>H$, the dynamics of $\Delta_{t}$ are described by (see Haber et al. (1999) [14])

$$
d \Delta_{t}= \begin{cases}d t & X_{t}<H \\ -\Delta_{t^{-}} & X_{t}=H \\ 0 & X_{t}>H\end{cases}
$$

where $\Delta_{t^{-}}$is the left limit of $\Delta_{t}$. The clock $\Delta_{t}$ is reset to zero when the barrier is reached from below, and does not change when the process is above the barrier. Default occurs only if the length of the current excursion exceeds the maximum time allowed in default, $\Delta_{t} \geq \Delta$, and the probability of default is $\mathbb{Q}\left[\Delta_{t} \geq \Delta\right]$.

As already observed, the triggering of the barrier is fairly robust, nevertheless, the approach based on Parisian times suffers from another drawback. As pointed out by Haber et al. (1999) [14] and Moraux (2003) [25], the resetting of the distress clock is still sensitive to short-term movements of the value process $X$, which makes the model non-suitable in at least the following cases. First, consider a distressed firm that exits bankruptcy before the grace period has elapsed. At the maturity of the debt the firm will meet its obligations, even if it has spent in financial distress a long time period. As a second case, consider a firm that experienced successive defaults, this without causing liquidation.

An alternative approach to the problem is to consider the cumulative excursion (or occupation) time instead of the continuous excursion time ${ }^{10}$. In such a way, one accounts for the whole past financial distress of the firm by considering the total time spent by the value process beyond the default threshold over the monitored time interval. This approach seems more suitable in order to study the financial history and distress periods of the debt issuer.

Formally, let $H$ denote the barrier, $T$ the maturity, and $\Delta$ the maximum duration of time allowed below the barrier, the distress clock (occupation time)

\footnotetext{
${ }^{9}$ Note that, if the process $X$ is currently above the default threshold, then $\Delta_{t}=0$.

${ }^{10}$ Financial options linked to cumulative excursions are called ParAsian options in Haber et al. (1999) [14] or occupation time derivatives in Hugonnier (1999) [17].
} 
is defined as follows

$$
\Delta_{0}^{T}=\int_{0}^{T} \mathbf{1}_{\left\{X_{u} \leq H\right\}} d u .
$$

$\Delta_{0}^{T}$ is a measure of the time the process $X$ spends below the barrier during the time interval $[0, T]$. The probability of default is defined by $\mathbb{Q}\left[\Delta_{0}^{T} \geq \Delta\right]=$ $\mathbb{E}\left[\mathbf{1}_{\left\{\Delta_{0}^{T} \geq \Delta\right\}}\right]$.

In this formulation, the clock is not reset to zero, and the default time is defined as the first instant the process $X$ has spent totally an amount of time $\Delta$ below the default boundary,

$$
\tau^{*}=\inf \left\{t \in[0, T]: \Delta_{0}^{t} \geq \Delta\right\} .
$$

The excursion approach, based on both Parisian and occupation times, can be generalized in several ways. Possible extensions of such a method are based on weighted excursions.

Let $\varphi$ be a non-negative function, bounded on $[0,+\infty) \times[0,+\infty)$. Consider, for simplicity, a constant boundary $H$. Define the distress clock

$$
\tilde{\Delta}_{0}^{T}=\int_{0}^{T} \varphi(u, T) \mathbf{1}_{\left\{X_{u} \leq H\right\}} d u .
$$

Note that, when $\varphi$ is constant (and equal to 1 ) we recover the definition of occupation time given above.

When, for instance,

$$
\varphi(u, t)=e^{-\gamma(t-u)} \quad u \leq t \quad \gamma \geq 0,
$$

$\tilde{\Delta}_{0}^{T}$ can be considered as a weighted occupation time, being $\varphi$ a weight function ${ }^{11}$.

A generalization of the weighted occupation time approach has been proposed by Galai et al. (2003) [12], in which past excursions "weight less" than the current excursion.

When

$$
\varphi(u, t)=e^{-\gamma(t-u)} \mathbf{1}_{\left\{\tau_{t}^{H} \leq u\right\}},
$$

where $\tau_{t}^{H}$ is defined as in (8), we obtain a weighted version of the Parisian time approach discussed above ${ }^{12}$, with

$$
\tilde{\Delta}_{0}^{t}=\int_{\tau_{t}^{H}}^{t} e^{-\gamma(t-u)} \mathbf{1}_{\left\{X_{u} \leq H\right\}} d u .
$$

In the weighted excursion time approach, the default time is defined as the following random time

$$
\tau^{*}=\inf \left\{t \in[0, T]: \tilde{\Delta}_{0}^{t} \geq \Delta\right\} .
$$

${ }^{11}$ More generally, one may consider $\varphi(u, t)=\exp \left\{-\int_{u}^{t} \gamma(s) d s\right\}$, being $\gamma$ a nonnegative function.

${ }^{12}$ Note that, when $\gamma=0$, we have exactly the Parisian time specification. 
Of course, we always have to define the event that triggers default at maturity in a consistent way. The default time will be the minimum between the (weighted) excursion time and $\tau_{T}$, where

$$
\tau_{T}=\left\{\begin{array}{cl}
T & \text { if } X_{T} \leq H_{T} \\
+\infty & \text { otherwise }
\end{array}\right.
$$

$H_{T}$ can coincide with the face value of the debt.

In general, one can use Monte Carlo simulation in order to compute an estimate of the average time of default and the default probability.

\section{$5 \quad$ Modeling the severity of the financial distress}

In the previous sections we considered alternative specifications of the event that triggers default: first-passage time approach, which seems more favorable to bondholders, and the excursion time approach which, allowing for a grace period, favors debtor's rights. Occupation time approach lies somewhere between the classical first-passage and the Parisian time approaches. Generalizations based on a weighted definition of the distress clock are also possible.

Nevertheless, all these approaches do not take into account the severity of the financial distress. Nardon (2005) [26] defines the default time as the first instant when a relevant process either stays continuously for a certain time period beyond the distress threshold, or reaches another lower barrier. The hitting of such a lower barrier signals severe financial distress, causing immediate default of the debtor. This specification of the default time generalizes both the Parisian and first-passage time definitions.

In order to model the severity of the financial distress one may, alternatively, weight more strongly large deviations below the default boundary. As suggested by Haber et al. (1999) [14], the speed at which the distress clock $\tilde{\Delta}_{0}^{T}$ changes could be proportional to the distance of the value process from the default barrier.

A model that takes into account the severity of the distress is based on the weighted shortfall, which is a measure of the area of excursion. The weighted cumulative shortfall over the time interval $[0, T]$ is defined by

$$
\tilde{\Delta}_{0}^{T}=\int_{0}^{T} e^{-\gamma(T-u)}\left(H-X_{u}\right)^{+} d u,
$$

which derives from considering $\varphi(u, t)=e^{-\gamma(t-u)}\left(H-X_{u}\right)$ (see Giesecke (2004) [13]). A weighted shortfall based on the definition of Parisian time may be considered as well by defining $\varphi$ in a proper way. $\gamma$ may be interpreted as decay factor (as defined by Galai et al. (2003) [12]) of the excursion.

Galai et al. (2003) [12] define the distress clock as follows

$$
\tilde{\Delta}_{0}^{t}=\int_{0}^{\tau_{t}^{H}} e^{-\beta(t-u)} f\left(X_{u}\right) d u+\int_{\tau_{t}^{H}}^{t} e^{-\gamma(t-u)} f\left(X_{u}\right) d u
$$


where $\beta \geq 0$ and $\gamma \geq 0$ are the decay factors for the past and last excursions, respectively, and the function $f\left(X_{t}\right)$ models the severity of the distress. The authors consider the function

$$
f\left(X_{t}\right)=e^{\alpha \frac{H_{t}-X_{t}}{H_{t}}} \mathbf{1}_{\left\{X_{t} \leq H_{t}\right\}},
$$

where $\alpha \geq 0$, and $H$ is a time dependent threshold.

This approach could be applied in conjunction with the definition of multiple boundaries that signal different levels of severity (see Nardon (2005) [26]) and different weights assigned to excursions in given regions.

\section{Simulation analysis}

In this section we examine the effects of different default time specifications on credit spreads structure. In particular, in the numerical experiments we adopt the model proposed by Saá-Requjo and Santa-Clara (1999) [30], and the model of Cox et al. (1985) [9] for the short term interest rate, which are briefly summarized in the Appendixes A and B. Some results of the simulation analysis are then presented.

In order to evaluate the default probability we used analytical formulas (in the first-passage case) and Monte Carlo simulation in all other cases. In the simulation, we generated 100000 paths (50000 antithetic) of the process $X$. We considered a time step equal to $\Delta t=1 / 250$ (which approximatively corresponds to daily observations of the process $X$ ) and bond maturities (in years) that range in the interval $[0,20]$. The default probability is simply approximated by the relative frequency of the default event.

Figure 1 shows the term structure of credit spreads, as the writedown and the solvency ratio vary, for different specifications of the default time. More precisely, within the framework of Saá-Requejo and Santa-Clara (1999) [30], we considered the first-passage time case (first two figures), the Parisian time case (second two figures), and the occupation time case (last two figures). The delay parameter is set at $\Delta=0.5$ (six months). In Figure 2 we propose a similar experiment, letting the solvency ratio vary. In Figure 3 we reported the results of the same experiment, where we considered the weighted distress clock both in the consecutive and cumulative excursion cases. Figure 4 depicts credit spreads when we account for the severity of the distress ${ }^{13}$. Two different values of the parameter $\gamma$ are considered.

In all cases, the obtained term structures of credit spreads present the typical shape which is common in structural credit risk models ${ }^{14}$. We notice that, the introduction of a delay in the timing of default has the effect of changing both

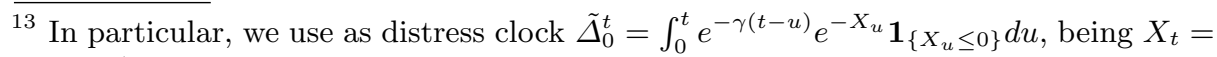
$\ln V_{t} / H_{t}$.

${ }^{14}$ Many authors argue that such term structures of default probabilities and credit spreads are not supported by empirical observations. In particular, the probability of default in reality is non-null as the time to maturity tends to zero. 

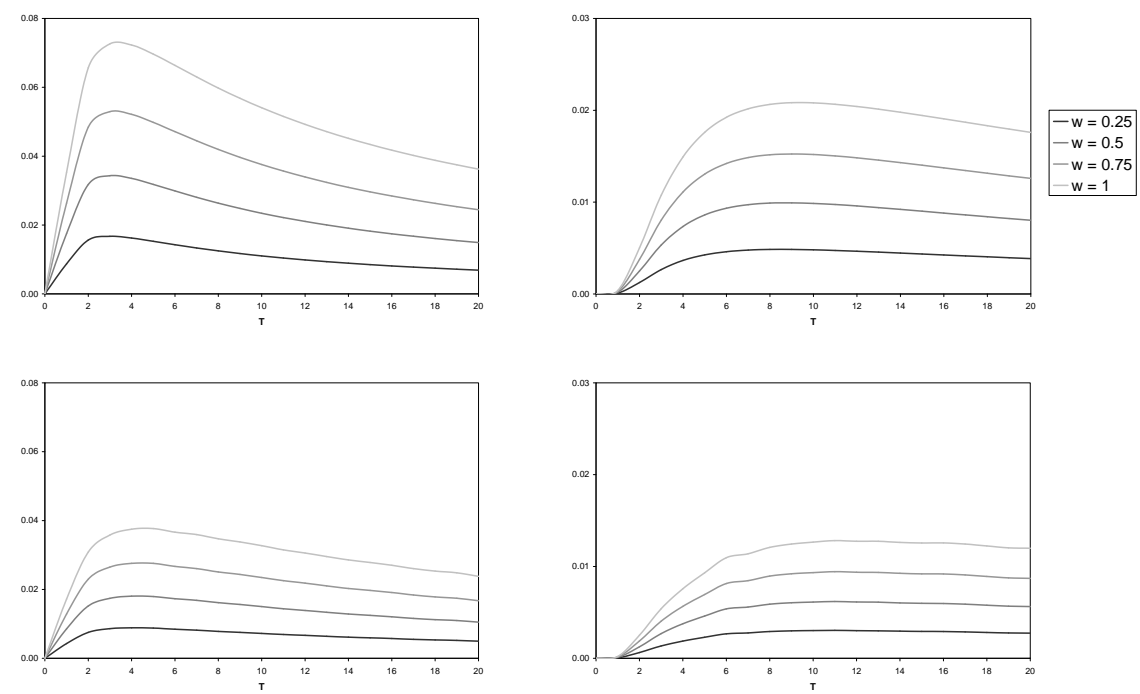

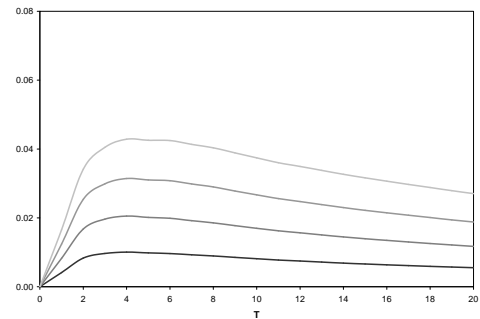

(a)

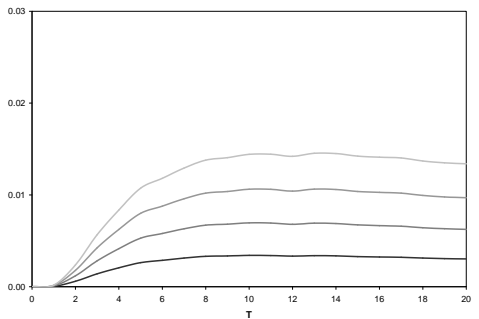

(b)

Fig. 1. Credit spreads structure for different values of the writedown $w \in$ $\{0.25,0.5,0.75,1\}$, in the first-passage, Parisian, and occupation time models, with $X_{0}=\ln 1.5$ in case (a) and $X_{0}=\ln 2$ in case (b). The other parameters are: $\mu=0.02$, $\sigma=0.2, \Delta=0.5, r_{0}=0.02, \theta=0.04, \kappa=0.5, \sigma_{r}=0.03$, and $\rho=0$.

the shape and level of credit spreads. In the model under consideration, when the barrier is set at zero, and being other model factors unchanged, as expected, we obtain higher bond values and consequently lower credit spreads. What is not highlighted by the pictures, is the fact that different hypothesis made on the events that triggers default, have an effect in the timing of default (intuitively, in the consecutive excursion approach the average default time is higher that in the cumulative excursion and, of course, in the first-passage approaches), and the probability of early default or default at maturity. 

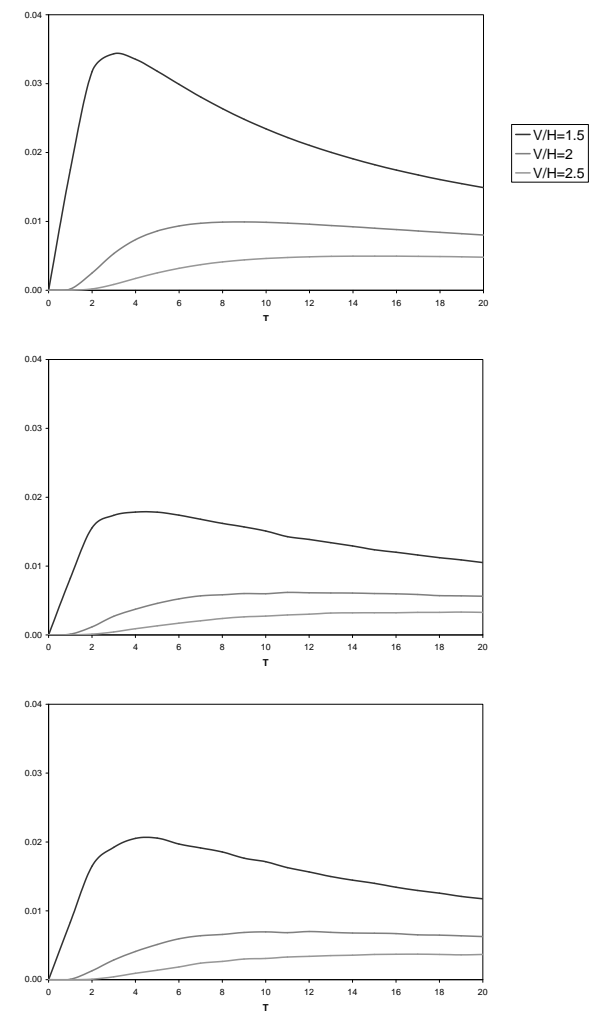

Fig. 2. Credit spreads structure for different values of the solvency ratio $V_{0} / H_{0} \in$ $\{1.5,2,2.5\}$, in the first-passage, Parisian, and occupation time models. The other parameters are: $\mu=0.02, \sigma=0.2, w=0.5, \Delta=0.5, r_{0}=0.02, \theta=0.04, \kappa=0.5$, $\sigma_{r}=0.03$, and $\rho=0$.

\section{Conclusions}

A number of approaches aim to capture the dynamics that lead to default. Excursion approach attempts to distinguish different credit events, such as the signaling of financial distress, or the filing for bankruptcy, from liquidation.

In this contribution, we considered a stylized model based on excursion times in order to evaluate defaultable debt. We then examined, through a simulation analysis, how different default time specifications affect credit spreads. We observed that, within the particular model we adopted, the introduction of a delay in the timing of default results in a significant decline of credit spreads.

As a final consideration, the approach based on excursions and related stopping times, is quite flexible and can be applied in conjunction with a rich class of models. A significant extension, left for future research, is the study of a model with endogenous default boundary. 


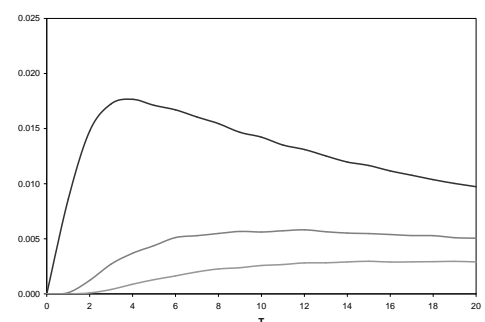

(a)

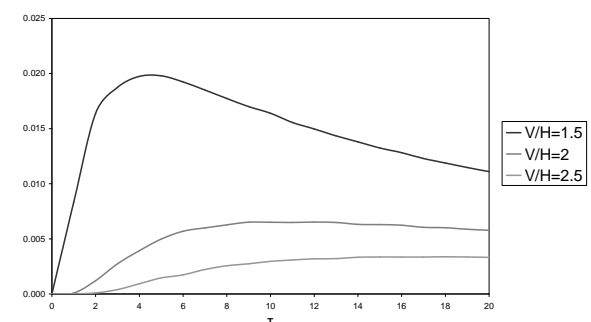

(b)

Fig. 3. Credit spreads structure for different values of the solvency ratio $V_{0} / H_{0} \in$ $\{1.5,2,2.5\}$, in the weighted Parisian time (a) and occupation time (b) approaches, with $\gamma=0.05$. The other parameters are: $\mu=0.02, \sigma=0.2, \Delta=0.5, r_{0}=0.02$, $\theta=0.04, \kappa=0.5, \sigma_{r}=0.03$, and $\rho=0$.

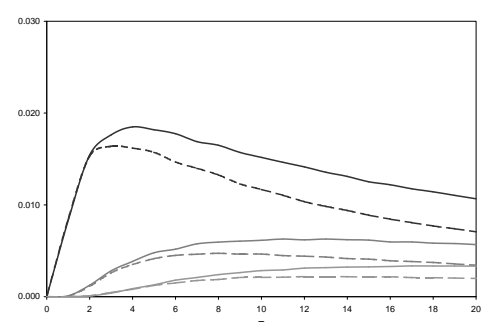

(a)

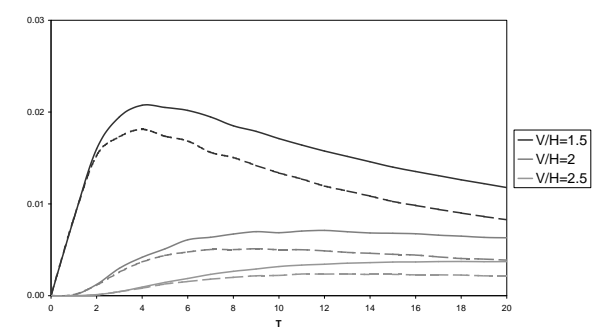

(b)

Fig. 4. Credit spreads structure for different values of the solvency ratio $V_{0} / H_{0} \in$ $\{1.5,2,2.5\}$, when the distress clock is defined as a weighted shortfall in the Parisian (a) and occupation time (b) cases, with $\gamma=0$ (solid line) and $\gamma=0.25$ (dashed line). The other parameters are: $\mu=0.02, \sigma=0.2, \Delta=0.5, r_{0}=0.02, \theta=0.04, \kappa=0.5$, $\sigma_{r}=0.03$, and $\rho=0$.

\section{Appendix A: Cox, Ingersoll and Ross (1985) model}

According to Cox, Ingersoll and Ross (1985) [9] (hereafter CIR), the default-free spot interest rate $r=\left(r_{t}\right)_{t \geq 0}$ is assumed to follow a mean reverting square root process. Its risk-adjusted dynamics is given by

$$
d r_{t}=\kappa\left(\theta-r_{t}\right) d t+\sigma_{r} \sqrt{r_{t}} d W_{t}^{r}
$$

where $r_{0}>0, \kappa, \theta$ and $\sigma_{r}>0$ are constant parameters, and $W^{r}$ is a standard Wiener process. The parameters $\theta$ and $\kappa$ are the long-term mean value of $r$ and the speed of adjustment to $\theta$, respectively.

Let $p(t, T)$ denote the price at time $t$ of a default-free ZCB with maturity date $T$. In CIR model, the value of the bond is given by

$$
p(t, T)=A(\tau) e^{-B(\tau) r_{t}},
$$


where $\tau=T-t$,

$$
\begin{aligned}
& A(\tau)=\left[\frac{2 \gamma e^{(\gamma+\kappa)^{\frac{\tau}{2}}}}{2 \gamma+(\gamma+\kappa)\left(e^{\gamma \tau}-1\right)}\right]^{2 \kappa \theta / \sigma_{r}^{2}}, \\
& B(\tau)=\frac{2\left(e^{\gamma \tau}-1\right)}{2 \gamma+(\gamma+\kappa)\left(e^{\gamma \tau}-1\right)},
\end{aligned}
$$

and $\gamma=\sqrt{\kappa^{2}+2 \sigma_{r}^{2}}$.

\section{Appendix B: Saá-Requejo and Santa-Clara (1999) model}

The model proposed by Saá-Requejo and Santa-Clara (1999) [30] is a generalization of the model of Nielsen et al. (1993) [27] and subsequently developed by Hsu et al. (2003) [16]. In these contributions, default-free interest rates are assumed stochastic.

We closely follow Saá-Requejo and Santa-Clara (1999) [30]. An important feature of the model under consideration is the definition of the default boundary $\left(H_{t}\right)$, which is a stochastic process. $H_{t}$ represents the total value at time $t$ of the liabilities (or part of them) in Saá-Requejo and Santa-Clara (1999) [30], the bankruptcy value of the firm in Hsu et al. (2003) [16].

As in Longstaff and Schwartz (1995) [23], the writedown $w$ is an exogenously given constant ${ }^{15}$. The recovery value upon default is then the quantity $1-w$ per units of face value of a default-free ZCB with maturity $T$. In particular, this assumption allows to valuing a security (even a complex liability) independently from other liabilities issued by the firm. A general assumption that is retained is the simultaneous default on all liabilities.

Let $v(t, T)$ denote the value at time $t$ of a defaultable ZCB with maturity at time T. $v(t, T)$ can be written as follows ${ }^{16}$ :

$$
\begin{aligned}
v(t, T) & =\mathbb{E}_{t}\left[\left(1-w \mathbf{1}_{\left\{\tau^{*} \leq T\right\}}\right) e^{-\int_{t}^{T} r(u) d u}\right] \\
& =p(t, T)-\mathbb{E}_{t}\left[w \mathbf{1}_{\left\{\tau^{*} \leq T\right\}} e^{-\int_{t}^{T} r(u) d u}\right],
\end{aligned}
$$

where $p(t, T)$ is the time $t$ value of a default-free $\mathrm{ZCB}, \tau^{*}$ is the time of default, and $r$ is the default-free instantaneous interest rate. $\mathbb{E}_{t}(\cdot)$ here means expectation (conditional to the information set at time $t$ ) under the risk neutral measure $\mathbb{Q}$. All the processes in the following are defined with respect to such a martingale measure.

Let $V$ denote the process that describes firm's assets value, which satisfies the following stochastic differential equation

$$
d V_{t}=\left(r_{t}-\delta_{V}\right) V_{t} d t+\sigma_{V} V_{t} d W_{t}^{V} \quad V_{0}>0,
$$

${ }_{15}$ As an alternative, $w(T)$ could be a random variable $\left(\mathcal{F}_{T}\right.$-measurable), not correlated with other processes of the model. When it is assumed constant, $w$ is a parameter that has to be estimated.

${ }^{16}$ At maturity one has $v(T, T)=1-w \mathbf{1}_{\left\{\tau^{*} \leq T\right\}}$. 
where $\sigma_{V}>0$, and $\delta_{V}$ is the payout rate to the firm's investors. $W^{V}$ is a standard Wiener precess.

The default-free interest rate uncertainty depends on a Wiener process $W^{r}$, which is assumed correlated with $W^{V}$, being $\rho_{r V}$ the correlation coefficient.

The stochastic default boundary $H=\left(H_{t}\right)_{t \in[0, T]}$ is modeled as follows

$$
\frac{d H_{t}}{H_{t}}=\left(r_{t}-\delta_{H}\right) d t+\sigma_{H r} d W_{t}^{r}+\sigma_{H V} d W_{t}^{V} \quad H_{0}>0,
$$

where $\sigma_{H r}$ and $\sigma_{H V}$ are two positive constants, $\delta_{H}$ is a payout rate to the firm's bondholders. $H$ is modeled as a joint diffusion process whose uncertainty depends on both $V$ and $r$.

In this setting, default occurs at the first instant the value process $V$ falls below the liabilities value $H$. The default time is then defined as the following hitting time

$$
\tau^{*}=\inf \left\{t \geq 0: V_{u}=H_{u}\right\} .
$$

It is convenient to define a new process $X$,

$$
X_{t} \equiv \ln \frac{V_{t}}{H_{t}}=\ln V_{t}-\ln H_{t},
$$

which allows us to write

$$
\tau^{*}=\inf \left\{t \geq 0: X_{u}=0\right\} .
$$

The default time is defined as the first instant at which the log-solvency ratio reaches level zero.

It can be shown that $X$, as defined by (31), satisfies the stochastic differential equation $^{17}$

$$
d X_{t}=\mu d t+\sigma d W_{t}^{X} \quad X_{0}>0
$$

with

$$
\begin{gathered}
\mu=\delta_{H}-\delta_{V}-\frac{1}{2}\left(\sigma_{V}^{2}-\left(\sigma_{H V}^{2}+\sigma_{H r}^{2}+2 \rho_{r V} \sigma_{H V} \sigma_{H r}\right)\right) \\
\sigma^{2}=\left(\sigma_{V}-\sigma_{H V}\right)^{2}+\sigma_{H r}^{2}-2 \rho_{r V}\left(\sigma_{V}-\sigma_{H V}\right) \sigma_{H r} .
\end{gathered}
$$

$W^{X}$ is a Wiener process, defined such that

$$
\sigma W_{t}^{X}=\left(\sigma_{V}-\sigma_{H V}\right) W_{t}^{V}-\sigma_{H r} W_{t}^{r}
$$

and correlated with the Wiener process $W^{r}$, with correlation coefficient

$$
\rho=\frac{\rho_{r V}\left(\sigma_{V}-\sigma_{H V}\right)-\sigma_{H r}}{\sigma} .
$$

In the special case $\rho=0$, default is independent of default-free interest rate; as a result, defaultable ZCBs can be evaluated in closed-form:

$$
v(t, T)=p(t, T)-w p(t, T) \mathbb{Q}_{t}\left(\tau^{*} \leq T\right),
$$

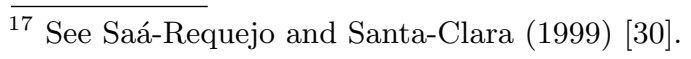


where the probability of default is given by ${ }^{18}$ :

$$
\mathbb{Q}_{t}\left(\tau^{*} \leq T\right)=\Phi\left(\frac{-X_{t}-\mu(T-t)}{\sigma \sqrt{T-t}}\right)+e^{-\frac{2 \mu X_{t}}{\sigma^{2}}} \Phi\left(\frac{-X_{t}+\mu(T-t)}{\sigma \sqrt{T-t}}\right) .
$$

If $\rho \neq 0$, no closed-form solutions are known ${ }^{19}$. If we adopt the model of Cox et al. (1985) [9] for $r$, when $\rho \neq 0$, we have to jointly consider the dynamics of the processes $r$ and $X$ :

$$
\begin{gathered}
d r_{t}=\left[\kappa\left(\theta-r_{t}\right)+\sigma_{r}^{2} B(\tau) r_{t}\right] d t+\sigma_{r} \sqrt{r_{t}} d W_{t}^{1}, \\
d X_{t}=\left[\mu+\rho \sigma \sigma_{r} \sqrt{r_{t}} B(\tau)\right] d t+\rho \sigma d W_{t}^{1}+\sqrt{1-\rho^{2}} \sigma d W_{t}^{2},
\end{gathered}
$$

where $W^{1}$ and $W^{2}$ are two independent Wiener processes. $B(\tau)$ is calculated using formula (26) in the CIR model.

In this case, the value of the defaultable ZCB is given by

$$
v(t, T)=p(t, T)-w p(t, T) \mathbb{Q}_{t}^{T}\left(\tau^{*} \leq T\right),
$$

where $\mathbb{Q}_{t}^{T}\left(\tau^{*} \leq T\right)$ is the forward risk adjusted probability of default (i.e. the probability measure under which asset values normalized by the price of a default-free ZCB with maturity $T$ are martingales).

\section{References}

1. Bielecki, T.R., Rutkowski, M.: Credit Risk: Modeling, Valuation and Hedging. Springer-Verlag, Berlin-Heidelberg (2002)

2. Black, F., Cox, J.C.: Valuing corporate securities: some effects of bond indeture provisions. Journal of Finance 31 (1976) 351-367

3. Black, F., Scholes M.: The pricing of options and corporate liabilities. Journal of Political Economy 81 (1973) 637-654

4. Briys, E., de Varenne, F.: Valuing fixed rate debt: An extention. Journal of Financial and Quantitative Analysis 32 (1997) 239-248

5. Broadie, M., Chernov, M., Sundaresan, S.: Optimal debt and equity values in the presence of Chapter 7 and Chapter 11. Working paper (2004)

6. Cathcart, L., El-Jahel, L.: Valuation of defaultable bonds. Journal of Fixed Income 8-1 (1998) 65-78

7. Chesney, M., Jeanblanc-Picqué, M., Yor, M.: Brownian excursions and Parisian barrier options. Advances in Applied Probability 29 (1997) 165-184

8. Collin-Dufresne, P., Goldstein, R.S.: Do credit spreads reflect stationary leverage ratios? Journal of Finance 56 (2001) 1929-1958

9. Cox, J.C., Ingersoll, J.E., Ross, S.: A theory of the term structure of interest rates. Econometrica 53 (1985) 385-407

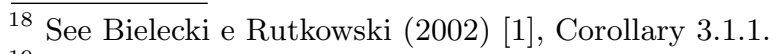

${ }^{19}$ Saá-Requejo and Santa-Clara (1999) [30] provide approximate solutions when default-free bond volatility is a deterministic function. In other cases, one need resort to computational techniques, such us Monte Carlo simulation. 
10. Eberhart, A.C., Altman, E.I., Aggarwal, R.: The equity performance of firms emerging from bankruptcy. Journal of Finance 54 (1999) 1855-1868

11. François, P., Morellec, E.: Capital structure and asset prices: some effetcs of bankruptcy procedures. Journal of Business 77-2 (2004) 387-411

12. Galai, D., Raviv, A., Wiener, Z.: Liquidation triggers and the valuation of equity and debt. Working paper (2003)

13. Giesecke, K.: Credit risk modeling and valuation: An introduction. Working paper, Cornell University (2004)

14. Haber, R.J., Schönbucher, P., Wilmott, P.: Pricing Parisian options. Journal of Derivatives 6-3 (1999) 71-79

15. Helwege, J.: How long do junk bonds spend in default? Journal of Finance 54-1 (1999) 341-357

16. Hsu, J.C., Saá-Requejo, J., Santa-Clara, P.: Bond pricing with default risk. Working paper (2003)

17. Hugonnier, J.: The Feynman-Kac formula and pricing occupation time derivatives. International Journal of Theoretical and Applied Finance 2-2 (1999) 153-178

18. Hui, C.H., Lo, C.F., Tsang, S.W.: Pricing corporate bond with dynamic default barriers. Journal of Risk 5-3 (2003) 17-39

19. Jarrow, R.A., Protter, P.: Structural versus reduced form models: a new information based perspective. Journal of Investment Management 2-2 (2004) 1-10

20. Kim, I.J., Ramaswamy, K., Sundaresan, S.: Does default risk coupons affect the valuation of corporate bonds? A contingent claims model. Financial Management 22-3 (1993) 117-131

21. Leland, H.E.: Corporate debt value, bond covenants, and optimal capital structure. Journal of Finance 59 (1994) 1213-1252

22. Leland, H.E., Toft, K.: Optimal capital structure, endogenous bankruptcy, and the term structure of credit spreads. Journal of Finance 51 (1996) 987-1019

23. Longstaff, F.A., Schwartz, E.S.: A simple approach to valuing risky fixed and floating rate debt. Journal of Fianance $\mathbf{5 0}$ (1995) 789-819

24. Merton, R.C.: On the pricing of corporate debt: the risk structure of interest rates. Journal of Finance 29 (1974) 449-470

25. Moraux, F.: Valuing corporate liabilities when the default threshold is not an absorbing barrier. Working paper, Université de Rennes I - IGR (2003)

26. Nardon M.: First passage and excursion time models for valuing defaultable bonds. Working paper, Department of Applied Mathematics, University Ca' Foscari of Venice (2005)

27. Nielsen, L.T., Saá-Requejo, J., Santa-Clara, P.: Default risk and interest rate risk: the term structure of default spreads. Working paper, INSEAD (1993)

28. Paseka, A.: Debt valuation with endogenous default and Chapter 11 reorganization. Working paper (2003)

29. Protter, P.: Stochastic Integration and Differential Equations. Springer-Verlag, New York (2004)

30. Saá-Requejo, J., Santa-Clara, P.: Bond pricing with default risk. Working paper (1999)

31. Shimko, D., Tejima, N., van Deventer, D.R.: The pricing of risky debt when interest rates are stochastic. Journal of Fixed Income 3-2 (1993) 58-65

32. Taurén, M.P.: A model of corporate bond prices with dynamic capital structure. Working paper (1999)

33. Yu, L.Z., Newton, D.P., Duck, P.W., Widdicks, M., Johnson, P.V.: Pricing credit risk as ParAsian options with endogenous recovery rate of corporate bonds. Working paper (2004) 